\title{
Solar Sail Equilibria in the Elliptical Restricted Three-Body Problem
}

\author{
Hexi Baoyin ${ }^{*}$ and Colin R. McInnes** \\ University of Strathclyde, Glasgow, G1 1XJ, Scotland, UK
}

\begin{abstract}
This paper investigates the existence and dynamical properties of artificial equilibria for solar sails in the elliptical restricted three-body problem. It is shown that planar two-dimensional equilibrium curves exist, embedded in three-dimensional space, in a non-uniformly rotating, pulsating coordinate system. Because of the stretching of the coordinate frame in the system plane, while the out-of-plane coordinate remains unstretched, three-dimensional equilibrium surfaces, which exist in the circular case, do not exist in the elliptical restricted three-body problem. Control in the neighborhood of an equilibrium point is investigated through a pole assignment scheme. This allows the possibility of practical out-of-plane equilibria in elliptical three-body systems with small eccentricity.
\end{abstract}

\section{Introduction}

The dynamics and control of solar sails in the circular restricted three-body problem have been investigated in some detail. ${ }^{1-3}$ The solar sail circular restricted three-body problem (luminous primary mass and a flat mirror) possesses infinite equilibrium surfaces parameterized by the solar sail lightness number, while the classical elliptical restricted three-body problem possesses only five Lagrange points. ${ }^{4}$ The so-called

\footnotetext{
${ }^{*}$ Visiting Scholar, Department of Mechanical Engineering, University of Strathclyde, Member AIAA. hexi.baoyin@strath.ac.uk

** Professor, Department of Mechanical Engineering, University of Strathclyde, Member AIAA.

colin.mcinnes@strath.ac.uk
} 
photo-gravitational circular restricted problem (luminous primary mass and a spherical test particle) has seven Lagrange points. ${ }^{5}$ The stability of these seven Lagrange points have been investigated by several authors for either the circular or elliptical restricted cases. ${ }^{6-11}$ In those papers instead of a solar sail, the term 'photogravitational' is adopted and is equivalent to the solar sail problem with the sail normal fixed along the Sun-line (spherical test particle). It can be shown that the collinear equilibrium points of the photo-gravitational elliptical restricted three-body problem are unstable, while the triangular points are Lyapunov stable in some parameter ranges.

Using the solar sail circular restricted three-body problem, various

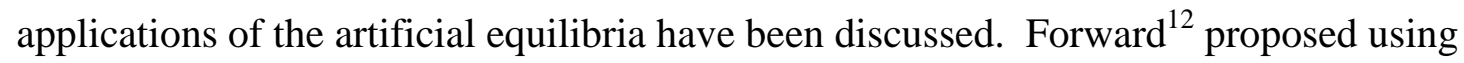
artificial equilibria high above the night-side of the Earth (equilibrium surfaces attached to the $\mathrm{L}_{2}$ point) to provide telecommunications services to high latitude users, while McInnes ${ }^{13,14}$ has proposed using artificial equilibria high above the day-side of the Earth (equilibrium surfaces attached to the $\mathrm{L}_{1}$ point) to provide continuous, realtime imaging of the poles. Morrow also proposed using three-body equilibrium surfaces to park solar sails in close proximity to asteroids, or other small solar system bodies. ${ }^{15}$ In-plane equilibria have also been considered as a useful location to station space weather missions, sunward to the classical $\mathrm{L}_{1}$ point. ${ }^{16}$

In this paper the solar sail elliptical restricted three-body problem will be investigated in some detail. Firstly, a dimensionless equation of motion will be derived using a non-uniformly rotating, pulsating coordinate system and the existence of planar equilibrium curves investigated. It will then be shown that the threedimensional equilibrium surfaces, which exist in the circular restricted three-body problem, do not exist for the elliptical restricted three-body problem. This is due to 
the stretching of the coordinate frame in the system plane, while the out-of-plane coordinate remains unstretched. Instead it is shown that planar two-dimensional equilibrium curves exist, embedded in three-dimensional space, in the non-uniformly rotating, pulsating coordinate system. The local stability of these equilibria will be studied numerically and it will be demonstrated that, in general, the equilibria are unstable. Finally, a control scheme for the stabilization of out-of-plane equilibria will be derived for the small eccentricity case. This analysis demonstrates the possibility of practical out-of-plane equilibria in small eccentricity systems, even although exact out-of-plane equilibria do not exist for the general elliptical restricted three-body problem. This is a key finding since out-of-plane equilibria in the Earth-Sun threebody system have been proposed for near-term applications of solar sailing. ${ }^{12-14}$

\section{Equations of Motion}

Firstly, we consider a planar elliptical restricted three-body system. It is assumed that an appropriate set of units is introduced so that the gravitational constant $G=1$, the semi-major axis of the problem $a=1$, and the system has unit total mass. Let $\mu$ be dimensionless mass of the smaller primary (Earth) and then $1-\mu$ will be the mass of the larger primary (Sun), which is luminous. As shown in Fig. 1, let $X, Y$ denote a fixed inertial coordinate frame, and using complex number notation define

$$
W=X+i Y
$$

Then, in a similar manner let $\xi, \eta$ denote a non-uniformly rotating coordinate frame where 


$$
\varsigma=\xi+i \eta
$$

Again, using complex number notation, the two reference frames can be connected through a rotation

$$
W=\varsigma e^{i f}
$$

where $f$ is true anomaly of the smaller primary in the elliptical three-body system, as shown in Fig. 1.

Using this notation, the equation of motion of a solar sail in the inertial coordinate system can be written by summing the gravitational acceleration of the two primary masses and the solar radiation pressure acceleration experienced by the solar sail to obtain

$$
\ddot{W}=-(1-\mu) \frac{W-W_{1}}{\tilde{r}_{1}^{3}}-\mu \frac{W-W_{2}}{\tilde{r}_{2}^{3}}+\beta(1-\mu) \frac{\cos ^{2} \alpha}{\tilde{r}_{1}^{2}} N
$$

where $\alpha$ is the angle between sail normal and the Sun-line, $N$ is sail unit normal vector, $W_{1}, W_{2}$ are coordinates of the Sun and Earth respectively while

$$
\begin{aligned}
& \tilde{r}_{1}=\sqrt{(\xi+\mu)^{2}+\eta^{2}} \\
& \tilde{r}_{2}=\sqrt{(\xi+\mu-1)^{2}+\eta^{2}}
\end{aligned}
$$


are the distances from the two primary masses to the solar sail. Again using complex notation, the sail unit normal vector can be written as

$$
N=N_{X}+i N_{Y}
$$

while the sail lightness number $\beta$ is defined as the ratio of the solar radiation pressure acceleration to the solar gravitational acceleration. ${ }^{1}$ From Eq. (3) the second time derivative of $W$ can be obtained as

$$
\ddot{W}=\left[\ddot{\varsigma}+2 i \dot{f} \dot{\varsigma}-\dot{f}^{2} \varsigma+i \ddot{f} \varsigma\right] e^{i f}
$$

Then, substituting Eq. (5) into Eq. (4), the equation of motion of the solar sail in the rotating coordinate frame can be written as

$$
\ddot{\varsigma}+2 i \dot{f} \dot{\varsigma}=-(1-\mu) \frac{\varsigma-\varsigma_{1}}{\tilde{r}_{1}^{3}}-\mu \frac{\varsigma-\varsigma_{2}}{\tilde{r}_{2}^{3}}+\beta(1-\mu) \frac{\cos ^{2} \alpha}{\tilde{r}_{1}^{2}} n+\varsigma \dot{f}^{2}-i \ddot{f} \varsigma
$$

where, $N=n e^{i f}$, with $n$ now defined in the rotating frame. The transformation between time and the true anomaly as independent variable can be written as

$$
\frac{d}{d t}=\frac{d f}{d t} \frac{d}{d f}
$$

In addition, a non-dimensional (complex) position coordinate can be defined as 


$$
w=\frac{\varsigma}{\tilde{r}}
$$

where $\tilde{r}$ is the distance between the two primaries, which can be obtained from the two-body problem and

$$
w=x+i y
$$

where $x$ and $y$ are the non-dimensional position variables of the solar sail in the rotating frame of reference. Since the distance between the two primaries is time varying, the rotating coordinate frame is now pulsating (stretched in a periodic manner). Using the pulsating coordinate system it can then be shown that

$$
\begin{aligned}
& \dot{\zeta}=\tilde{r} \dot{w}+w \dot{\widetilde{r}} \\
& \ddot{\zeta}=\tilde{r} \ddot{w}+2 \dot{\widetilde{r}} \dot{w}+w \dot{\widetilde{r}}
\end{aligned}
$$

and so by substituting Eqs. (7-10) into Eq. (6) it is found that

$$
\begin{aligned}
\tilde{r}^{2}\left(w^{\prime \prime}\right. & \left.+2 i w^{\prime}\right)+z\left(\ddot{\tilde{r}}-\tilde{r} \dot{f}^{2}\right)+\left(w^{\prime}+i w\right)(\tilde{r} \ddot{f}+2 \dot{\tilde{r}} \tilde{f}) \\
& =-(1-\mu) \frac{w-w_{1}}{\tilde{r}^{2} r_{1}^{3}}-\mu \frac{w-w_{2}}{\tilde{r}^{2} r_{2}^{3}}+\beta(1-\mu) \frac{\cos ^{2} \alpha}{\tilde{r}^{2} r_{1}^{2}} n
\end{aligned}
$$

where $r_{1}=\tilde{r}_{1} / \tilde{r}, r_{2}=\tilde{r}_{2} / \tilde{r}$ and the prime indicates derivatives with respect to true anomaly $f$. Considering the two-body orbit of the primaries to determine the time derivative terms yields ${ }^{4}$ 


$$
w^{\prime \prime}+2 i w^{\prime}=\frac{1}{1+e \cos f}\left[w-(1-\mu) \frac{w-w_{1}}{r_{1}^{3}}-\mu \frac{w-w_{2}}{r_{2}^{3}}+\beta(1-\mu) \frac{\cos ^{2} \alpha}{r_{1}^{2}} n\right]
$$

which can be re-written in real number form as

$$
\begin{aligned}
& \left(x^{\prime \prime}-2 y^{\prime}\right)=\frac{1}{(1+e \cos f)}\left[\frac{\partial \Omega}{\partial x}+\beta(1-\mu) \frac{\cos ^{2} \alpha}{r_{1}^{2}} n_{x}\right] \\
& \left(y^{\prime \prime}+2 x^{\prime}\right)=\frac{1}{(1+e \cos f)}\left[\frac{\partial \Omega}{\partial y}+\beta(1-\mu) \frac{\cos ^{2} \alpha}{r_{1}^{2}} n_{y}\right]
\end{aligned}
$$

where the effective potential of the problem can be written as

$$
\Omega=\frac{1}{2}\left(x^{2}+y^{2}\right)+\frac{1-\mu}{r_{1}}+\frac{\mu}{r_{2}}
$$

As a more compact form Eqs. (13) can be written in vector form as

$$
\mathbf{s}^{\prime \prime}+S \mathbf{s}^{\prime}=\frac{1}{(1+e \cos f)}[\nabla \Omega+\mathbf{a}]
$$

where $\mathbf{s}=x \mathbf{i}+y \mathbf{j}$ for unit vectors $\mathbf{i}$ and $\mathbf{j}$ along the $x$ and $y$ axes in the rotating coordinate system, $S=\left[\begin{array}{cc}0 & -2 \\ 2 & 0\end{array}\right]$, and

$$
\mathbf{a}=\beta \frac{1-\mu}{r_{1}^{2}} \frac{\left(\mathbf{r}_{1} \cdot \mathbf{n}\right)^{2}}{r_{1}^{2}} \mathbf{n}
$$


where the sail unit normal vector is now written as $\mathbf{n}=n_{x} \mathbf{i}+n_{y} \mathbf{j}$. The equation of motion in the non-uniformly rotating, pulsating coordinate system will now be used to investigate the existence of equilibria in the solar sail elliptical restricted three-body problem.

\section{Equilibrium Solutions}

In order to determine if equilibria exist in the solar sail elliptical restricted three-body problem, the equilibrium conditions $x^{\prime \prime}=y^{\prime \prime}=x^{\prime}=y^{\prime}=0$ are substituted into Eq. (14). Then, equilibrium solutions must satisfy the identity

$$
\nabla \Omega+\mathbf{a}=0
$$

Taking the vector product, and scalar product of $\mathbf{n}$ on Eq. (16) it follows that

$$
\begin{gathered}
\nabla \Omega \times \mathbf{n}=0 \Rightarrow \mathbf{n}=\frac{\nabla \Omega}{|\nabla \Omega|} \\
\beta=\frac{1}{(1-\mu)} \frac{(\nabla \Omega \cdot \mathbf{n}) r_{1}^{4}}{\left(\mathbf{r}_{1} \cdot \mathbf{n}\right)^{2}}
\end{gathered}
$$

Equation (17a) implies that at an equilibrium point the sail normal is always directed opposite to the sum of the gravitational force from the primary masses and the centripetal force acting on the solar sail, while Eq. (17b) can be used to determined the required sail lightness number for equilibrium. In addition, because the condition for equilibrium for the elliptical case (in the rotating, pulsating coordinate frame) has 
a similar form to the circular case, ${ }^{1,2}$ it is easy to see that planar equilibrium curves in the non-uniformly rotating, pulsating coordinate frame are similar to the equilibrium curves in system plane of the circular case discussed in Ref. 1 and 2. Far from the Earth the planar equilibrium curves are near circular. However, there is a more complex topology near the Earth, with allowed and forbidden areas, as shown in Fig. 2. Because the coordinate systems are different in the elliptical and circular cases, there is a significant difference between the two families of equilibrium curves. While the planar equilibrium solution curves of the circular case are invariant in a uniformly rotating coordinate frame, the curves of the elliptical case are deforming as the primaries orbit each other, just as the distance between the classical Lagrange points and primaries varies in the elliptical restricted three-body problem. ${ }^{4}$

Figs. 3 show the equilibrium curves in a non-pulsing coordinate frame for Earth, where $f=0$, and (a) is far Earth case and (b) is near Earth case. Figs. 4 show the equilibrium curves near Mercury, where (a) $f=0$, (b) $f=\frac{\pi}{2}$, and (c) $f=\pi$. Due to the large eccentricity of Mercury, the curves change significantly both in position and size (note that here the dimensionless distance is based on the semimajor axis of Earth's orbit). System parameters are taken as $\mu=3.04036 \times 10^{-6}$, $e=0.0167$ for Earth, and $\mu=1.672 \times 10^{-7}, e=0.206$ for Mercury. The figures indicate that the position and size of the equilibrium curves are varying in a nonpulsing coordinate frame, although they are not varying in the pulsing coordinate frame.

Finally, the three-dimensional case will be considered. Using the above analysis, it can be shown that the dimensionless equation of the motion can be written as 


$$
\begin{aligned}
& \left(x^{\prime \prime}-2 y^{\prime}\right)=\frac{1}{(1+e \cos f)}\left[\frac{\partial \Omega}{\partial x}+\beta(1-\mu) \frac{\cos ^{2} \alpha}{r_{1}^{2}} n_{x}\right] \\
& \left(y^{\prime \prime}+2 x^{\prime}\right)=\frac{1}{(1+e \cos f)}\left[\frac{\partial \Omega}{\partial y}+\beta(1-\mu) \frac{\cos ^{2} \alpha}{r_{1}^{2}} n_{y}\right] \\
& \left(z^{\prime \prime}+z\right)=\frac{1}{(1+e \cos f)}\left[\frac{\partial \Omega}{\partial z}+\beta(1-\mu) \frac{\cos ^{2} \alpha}{r_{1}^{2}} n_{z}\right]
\end{aligned}
$$

where

$$
\begin{aligned}
& \Omega=\frac{1}{2}\left(x^{2}+y^{2}+z^{2}\right)+\frac{1-\mu}{r_{1}}+\frac{\mu}{r_{2}} \\
& r_{1}=\sqrt{(x+\mu)^{2}+y^{2}+z^{2}} \\
& r_{2}=\sqrt{(x+\mu-1)^{2}+y^{2}+z^{2}}
\end{aligned}
$$

Because of the term $(1+e \cos f) z$ in Eq. (18c) when the equilibrium condition is imposed, in the three-dimensional case there are no three-dimensional equilibrium surfaces in the non-uniformly rotating, pulsating coordinate frame. Therefore, for the solar sail elliptical restricted three-body problem, equilibrium solutions only exist in the plane of the system as two-dimensional planar curves embedded in threedimensional space. This is due to the stretching of the coordinate frame in the system plane, while the out-of-plane coordinate remains unstretched.

Given that out-of-plane equilibria in the Sun-Earth restricted three-body problem have been proposed as a near-term application of solar sailing ${ }^{12-14}$ this result appears to be problematic. However, as will be seen later, active control can be used 
to establish practical equilibria for the small eccentricity case. Since the eccentricity of the Earth's orbit $e=0.0167$, this ensures that applications of out-of-plane equilibria are still in principle possible.

\section{Stability of Equilibria}

In order to determine the local stability of the equilibrium points in the linear sense, we apply an infinitesimal perturbation $\boldsymbol{\delta}$ to an equilibrium point, and so the variation of Eq. (14) can be obtained as

$$
\boldsymbol{\delta}^{\prime \prime}+S \boldsymbol{\delta}^{\prime \prime}-\frac{1}{(1+e \cos f)}\left(\frac{\partial(\nabla \Omega)}{\partial \mathbf{r}}+\frac{\partial \mathbf{a}}{\partial \mathbf{r}}\right) \boldsymbol{\delta}=0
$$

where

$$
\frac{\partial \mathbf{a}}{\partial \mathbf{r}}=\frac{4 \nabla \Omega \mathbf{r}_{1}^{T}}{\left(\mathbf{r}_{1} \cdot \mathbf{r}_{1}\right)}-\frac{2 \nabla \Omega \nabla \Omega^{T}}{\left(\mathbf{r}_{1} \cdot \nabla \Omega\right)}
$$

and matrix $K=\left(\frac{\partial(\nabla \Omega)}{\partial \mathbf{r}}+\frac{\partial \mathbf{a}}{\partial \mathbf{r}}\right)$ has to be evaluated at the equilibrium point. Note that in Eq. (20) it is assumed that the direction of the sail normal vector is fixed as the infinitesimal perturbation is applied to the position variable. In addition, since the matrix $K$ is time-dependent, the problem at hand becomes the stability of a linear system with time-dependent coefficients. To study the stability, Eq. (19) can be represented in the form of first order differential equations as

$$
\chi^{\prime}=A \chi
$$


where, $\chi=\left[\boldsymbol{\delta}, \boldsymbol{\delta}^{\prime}\right]^{T}$ and the system matrix $A$ is defined as

$$
A=\left[\begin{array}{cc}
0 & I \\
K /(1+e \cos f) & -S
\end{array}\right]
$$

where $I$ is a unit matrix. Since the system has time-dependent coefficients, its stability cannot be directly determined by the eigenvalues of the matrix $A$. However because $A(f)=A(f+2 \pi)$, according to Floquet theory, the stability of a periodic coefficient linear system can be determined by the system behavior over one period. The basic idea is first to define a matrix $Q(f)$ by

$$
Q^{\prime}(f)=A(f) Q(f)
$$

where $Q(0)=I$ is an appropriate dimensional unit matrix. Then, Eq. (22) is integrated to obtain $Q(2 \pi)$. If the eigenvalues $\lambda_{i}$ of matrix $Q(2 \pi)$ satisfy $\left|\lambda_{i}\right| \leq 1$, then the system is stable, otherwise it is unstable. Unfortunately, because the matrix $A(f)$ cannot commute with its integral matrix $\int_{0}^{f} A(\tau) d \tau$, we cannot integrate Eq. (22) to get the closed form solution of $Q(2 \pi)$, but numerical integration is always available.

As a trial, we have investigated the stability of equilibria in some areas, defined by the shaded area of Fig. 2, using numerical integration, but only with the parameters $\mu=3.04036 \times 10^{-6}$ and $e=0.0167$ for the Earth-Sun elliptical restricted three-body problem. Numerical results show that those equilibria in the shaded area 
are all unstable, as expected. Indeed, we expect all equilibria for the solar sail elliptical restricted three-body problem to be unstable, other than the classical triangular points for some parameter ranges. ${ }^{7}$

\section{Control of Out-of-Plane Equilibria}

In order to enable unstable equilibria to be used for practical applications, active control is clearly required. In addition, in order to allow out-of-plane equilibria to be used in the small eccentricity case, active control will again be required. Here we consider orbit control manipulated with active control of the sail attitude.

Usually, a nonlinear system is linearized around an equilibrium point and controlled in a local neighborhood of it. However, in the solar sail elliptical restricted three-body problem it has been shown that out-of-plane equilibria do not exist. In the case $e<<1$, if the term $e \cos f$ is ignored in Eqs. (18), we can obtain an approximated equilibrium point which is positioned in the pulsing coordinate in a similar manner to the circular case (since the out-of-plane axis is not pulsating).

To consider linear control around an approximated equilibrium point, we assume that Eqs. (18) are linearized around the approximated equilibrium point $(e<<1)$, and the sail attitude is used as the control variable. Then, the controlled equation can be written as

$$
\chi^{\prime}=A \chi+B \mathbf{u}
$$

where, $\boldsymbol{\chi}=\left[x, y, z, x^{\prime}, y^{\prime}, z^{\prime}\right]^{T}$ and 


$$
\begin{aligned}
& A=\left[\begin{array}{cc}
0 & I \\
K /(1+e \cos f)-\bar{K} & -\bar{S}
\end{array}\right] \\
& \bar{K}=\left[\begin{array}{lll}
0 & 0 & 0 \\
0 & 0 & 0 \\
0 & 0 & 1
\end{array}\right] \\
& \bar{S}=\left[\begin{array}{ccc}
0 & -2 & 0 \\
2 & 0 & 0 \\
0 & 0 & 0
\end{array}\right] \\
& B=\left[\begin{array}{ll}
\mathbf{0} & \frac{\partial \mathbf{a}}{\partial \mathbf{n}}
\end{array}\right]_{3 \times 6}^{T} \\
& \frac{\partial \mathbf{a}}{\partial \mathbf{n}}=|\nabla \Omega| I+\frac{2|\nabla \Omega|}{\left(\nabla \Omega \cdot \mathbf{r}_{1}\right)} \nabla \Omega \mathbf{r}_{1}^{T}
\end{aligned}
$$

where $\mathbf{n}=\left[n_{x}, n_{y}, n_{z}\right]^{T}, \mathbf{u}=\delta \mathbf{n}$ and

This is a control problem of a time-varying linear system, because both $A$ and $B$ are time-dependent matrices. Therefore, making use of standard time-varying linearquadratic theory, ${ }^{17}$ one can design an optimal control law for the system. Below, however, we provide a simpler but non-optimal control design for the sail control in the $x z$-plane of the problem, where the equilibria of practical interest are located.

Since the eccentricity of the Earth's orbit $e \approx 0.0167$ a simple feedback control law can be designed. First, we design the feedback gains for the system 


$$
\chi^{\prime}=A_{0} \chi+B_{0} \mathbf{u}
$$

where matrices $A_{0}$ and $B_{0}$ are obtained by substituting $e=0$ into matrices $A$ and $B$ of Eq. (24) and so a full state feedback control is defined by

$$
\mathbf{u}=G \chi
$$

where the gain matrix $G$ will be obtained by pole assignment. After a control scheme is designed, the stability of the controlled system has to be numerically checked by using Floquet theory discussed above, because these gains are not exactly designed for the original system, defined by Eq. (24), but for the approximate system, defined by Eq. (26). Here an example is given to verify the possibility of solar sail control around an approximated equilibrium point in $x z$-plane of the Sun-Earth elliptical restricted three-body problem. The required equilibrium point is defined as $x=0.9, y=0, z=0.1$ which requires a sail lightness number $\beta=0.304779$. Then, if the desired poles are defined as $-5 \pm 2 i,-5 \pm 3 i,-6 \pm 3 i$ the feedback gain matrix $G$ is obtained as

$$
G=\left[\begin{array}{cccccc}
5.2520 & 0.0000 & 0.6402 & 2.1416 & 2.1416 & -0.4160 \\
0.0000 & 13.6908 & -0.0000 & -5.8857 & 11.7714 & -0.0000 \\
-3.5342 & -0.0000 & -0.4522 & -1.6177 & -1.6177 & 5.7060
\end{array}\right]
$$

The behavior of the controlled time-varying system (Eq. (24)) with feedback gains $G$, which is obtained by the approximated system (Eq. (26)) is shown in Fig. 5. It can be 
seen that the out-of-plane equiliria is completely controlled by the linear feedback scheme in the Sun-Earth elliptical restricted three-body problem.

\section{Conclusions}

This paper has investigated equilibria in the solar sail elliptical restricted three-body problem, and the following results obtained: There are no equilibrium surfaces in the three-dimensional, elliptical restricted three-body problem, but two-dimensional planar equilibrium curves do exist in the plane of the system, with the curve shapes in the non-uniformly rotating, pulsating system the same as the circular case; These equilibrium points are unstable; Although out-of-plane equilibrium points do not exist, when $e<<1$ the sail can be controlled around approximated equilibrium points. Therefore, solar sails can still be utilized for practical applications at out-of-plane equilibria of the Sun-Earth elliptical restricted three-body problem.

\section{References}

${ }^{1}$ McInnes, C. R., Solar Sailing: Technology, Dynamics and Mission Applications, Springer-Praxis, pp. 214-222, 1999.

${ }^{2}$ McInnes, C. R., Mcdonald, A. J. C., Simmons, J. F. L. and MacDonald, E. W., "Solar Sail Parking in Restricted Three-Body System,” Journal of Guidance, Control, and Dynamics, Vol.17, No. 2, 1994, pp. 399-406.

${ }^{3}$ McInnes, C.R., “Artificial Lagrange Points for a Non-Perfect Solar Sail,” Journal of Guidance, Control and Dynamics, Vol. 22, No. 1, 1999, pp. 185-187.

${ }^{4}$ Szebehely, V., Theory of Orbits: the Restricted Problem of Three Bodies, Academic Press, New York, pp. 588-594, 1967. 
${ }^{5}$ Simmons, J.F.L., McDonald, A.J.C., and Brown, J.C., “The Restricted Three-Body Problem with Radiation Pressure,” Celestial Mechanics, Vol. 35, No. 2, 1985, pp. 145-187.

${ }^{6}$ Perezhogin, A. A., "Stability of the Sixth and Seventh Libration Points in the Photogravitational Restricted Circular Three-Body Problem”, Soviet Astronomy Letters, Vol. 2, No. 5, pp. 174-175, 1976.

${ }^{7}$ Markellos, V. V., Perdios, E., and Labropoulou, P., “Linear Stability of the Triangular Equilibrium points in the Photogravitational Elliptic Restricted Problem, I”, Astrophysics and Space Science, Vol. 194, pp. 207-213, 1992.

${ }^{8}$ Markellos, V. V., Perdios, E., and Georghiou, C., "Linear Stability of the Triangular Equilibrium Points in the Photogravitational Elliptic Restricted Problem, II”, Astrophysics and Space Science, Vol. 199, pp. 23-33, 1993.

${ }^{9}$ Sahoo, S. K., and Ishwar, B., "Stability of Collinear Equilibrium Points in the Generalized Photogravitational Elliptic Restricted Three-Body Problem”, Bulletin of the Astronomical Society of India, Vol. 28, pp. 579-586, 2000.

${ }^{10}$ Singh, J., and Ishwar, B., "Stability of Triangular points in the generalized photogravitational elliptic restricted three-body problem”, Bulletin of the Astronomical Society of India, Vol. 27, pp. 415-424, 1999

${ }^{11}$ Kunitsyn, A. L., and Tureshbaev, A. T., "Stability of the triangular libration points in the photogravitational three-bpdy problem”, Soviet Astronomy Letters, Vol. 11, No.1, pp.60-62, 1985.

${ }^{12}$ Forward, R.L., “The Statite: A Spacecraft That Does Not Orbit," Journal of Spacecraft and Rockets, Vol. 28, No. 5, 1991, pp. 606-611.

${ }^{13}$ McInnes, C.R., “Solar Sailing: Orbital Mechanics and Mission Applications,” Advances in Space Research, Vol. 31, No. 8, pp. 1971-1980, 2003. 
${ }^{14}$ McInnes, C.R., "Solar Sailing: Mission Applications and Engineering Challenges,” Philosophical Transactions of the Royal Society A, Vol. 361, No. 1813, pp. 29893008, 2003.

${ }^{15}$ Morrow, E., Scherees, D.J., and Lubin, D., "Solar Sail Operations at Asteroids," Journal of Spacecraft and Rockets, Vol. 38, No. 2, pp. 279-286, 2001.

${ }^{16}$ West, J., "Solar Sail Vehicle System Design for the Geostorm Warning Mission,” AIAA-2000-5326, Space 2000 Conference and Exposition , September 19-21, 2000.

${ }^{17}$ Afanasev, V. N., Kolmanovskii, V. B., and Nosov, V. R., Mathematical Theory of Control System Design, Kluwer Academic Publishers, 1996. 


\section{Figures Captions}

Figure 1. Non-uniformly rotating, pulsating coordinate system

Figure 2. Region of existence of equilibrium solutions and stability areas analyzed

Figures 3. Equilibrium solution curves for Earth $(f=0)$

(a) far from Earth (1: $\beta=0.1,2: \beta=0.5,3: \beta=0.7,4: \beta=0.9$ )

(b) vicinity of Earth (1: $\beta=0.04,2: \beta=0.1,3: \beta=0.3,4: \beta=0.9$ )

Figures 4. Equilibrium solution curves in the vicinity of Mercury (1: $\beta=0.04,2$ :

$\beta=0.1,3: \beta=0.3,4: \beta=0.9$ )
(a) $f=0$;
(b) $f=\frac{\pi}{2}$;
(c) $f=\pi$

Figure 5. Behavior of controlled time-varying linear system 
Figure 1.

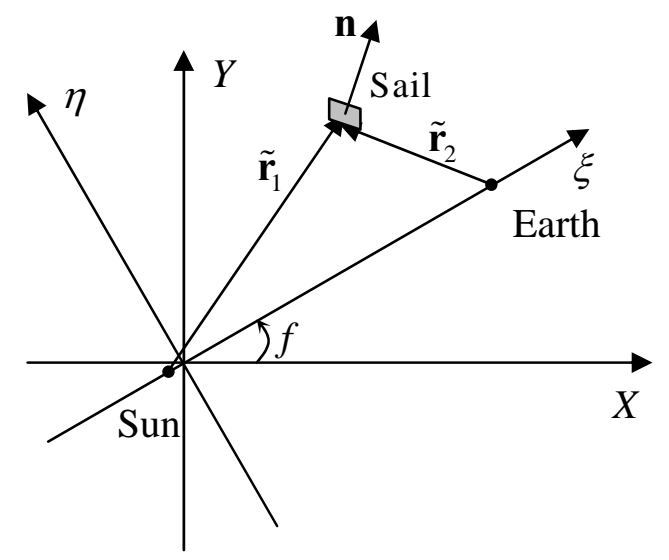


Figure 2

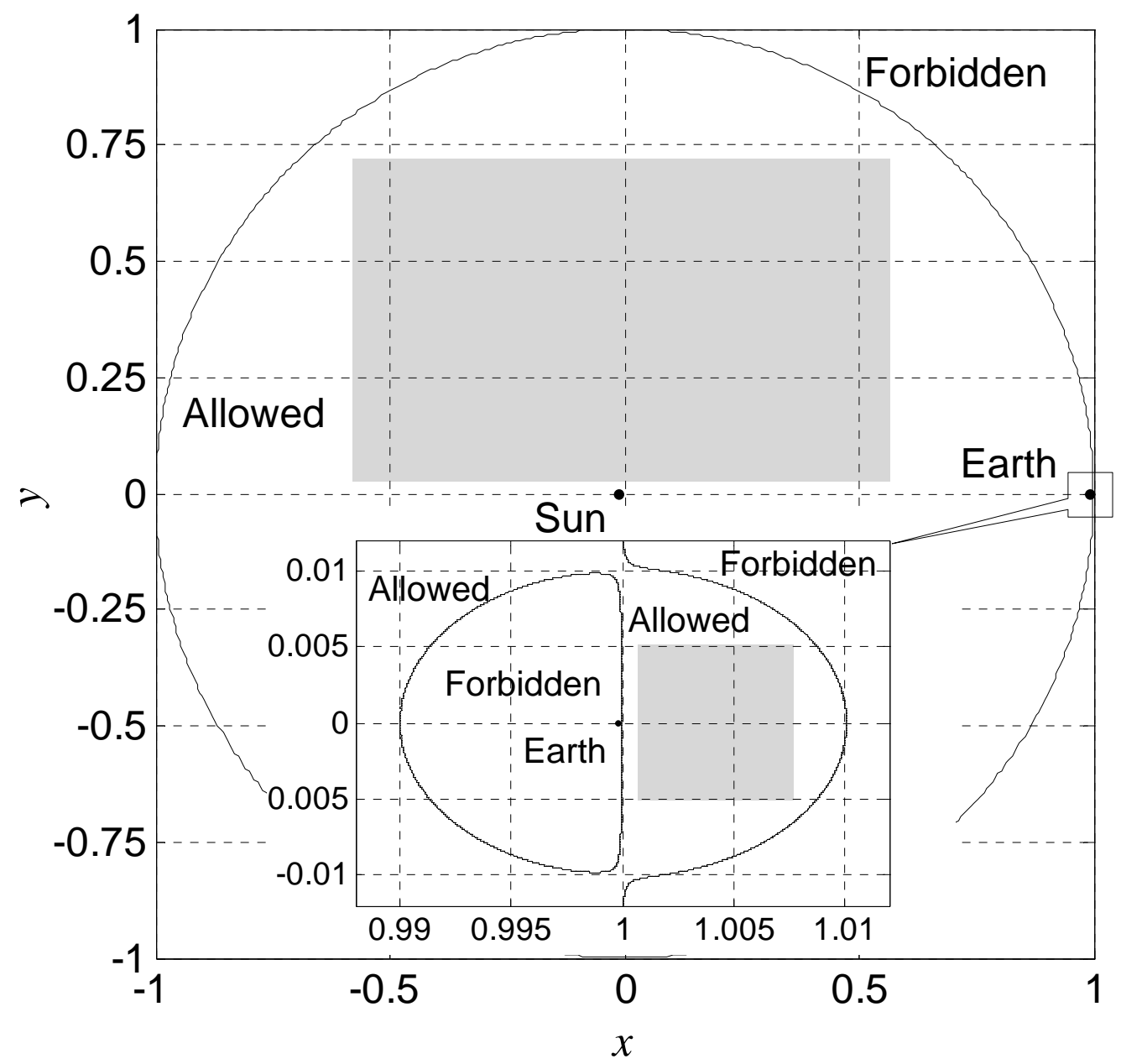


Figure 3 (a).

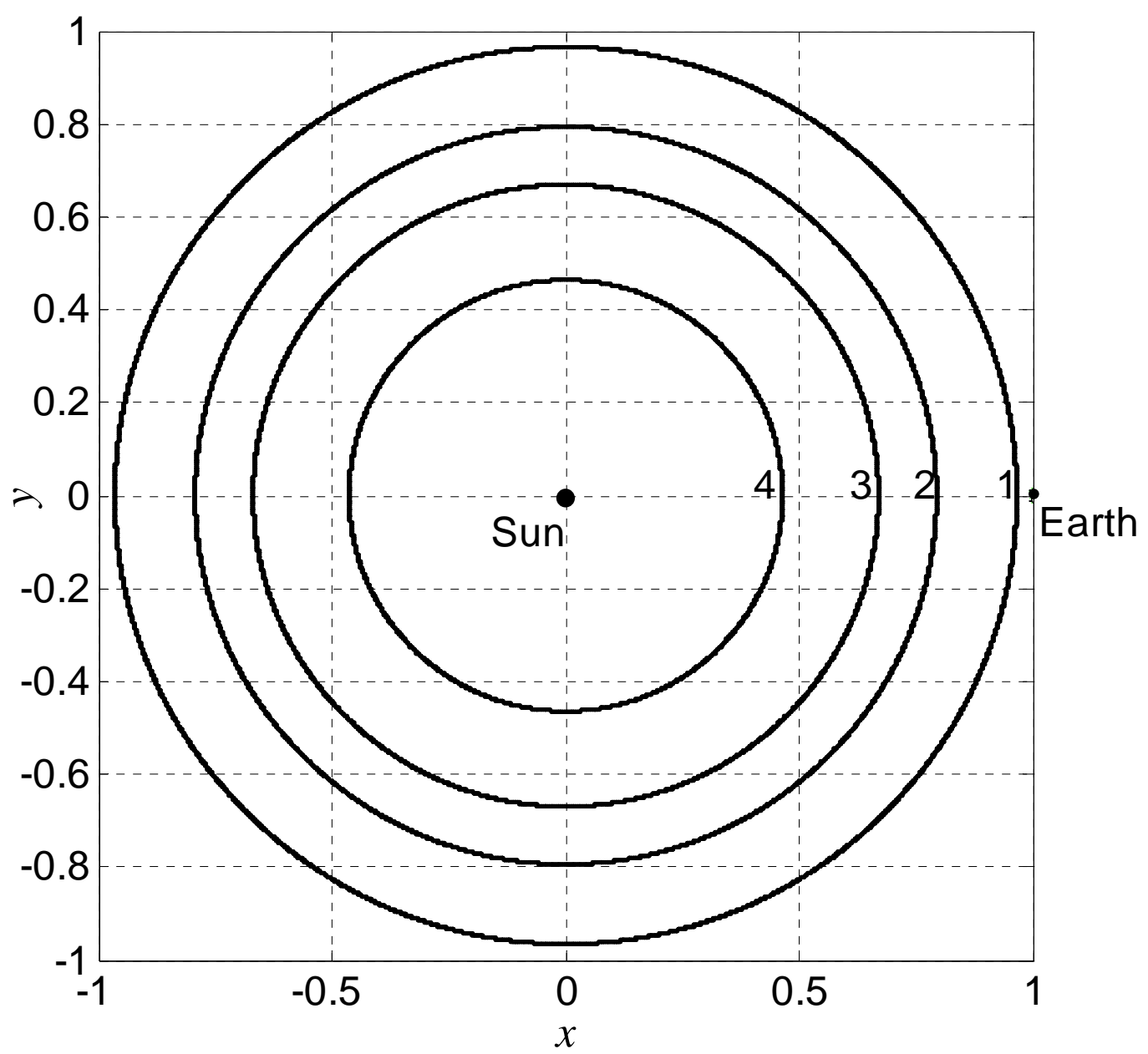


Figure 3 (b).

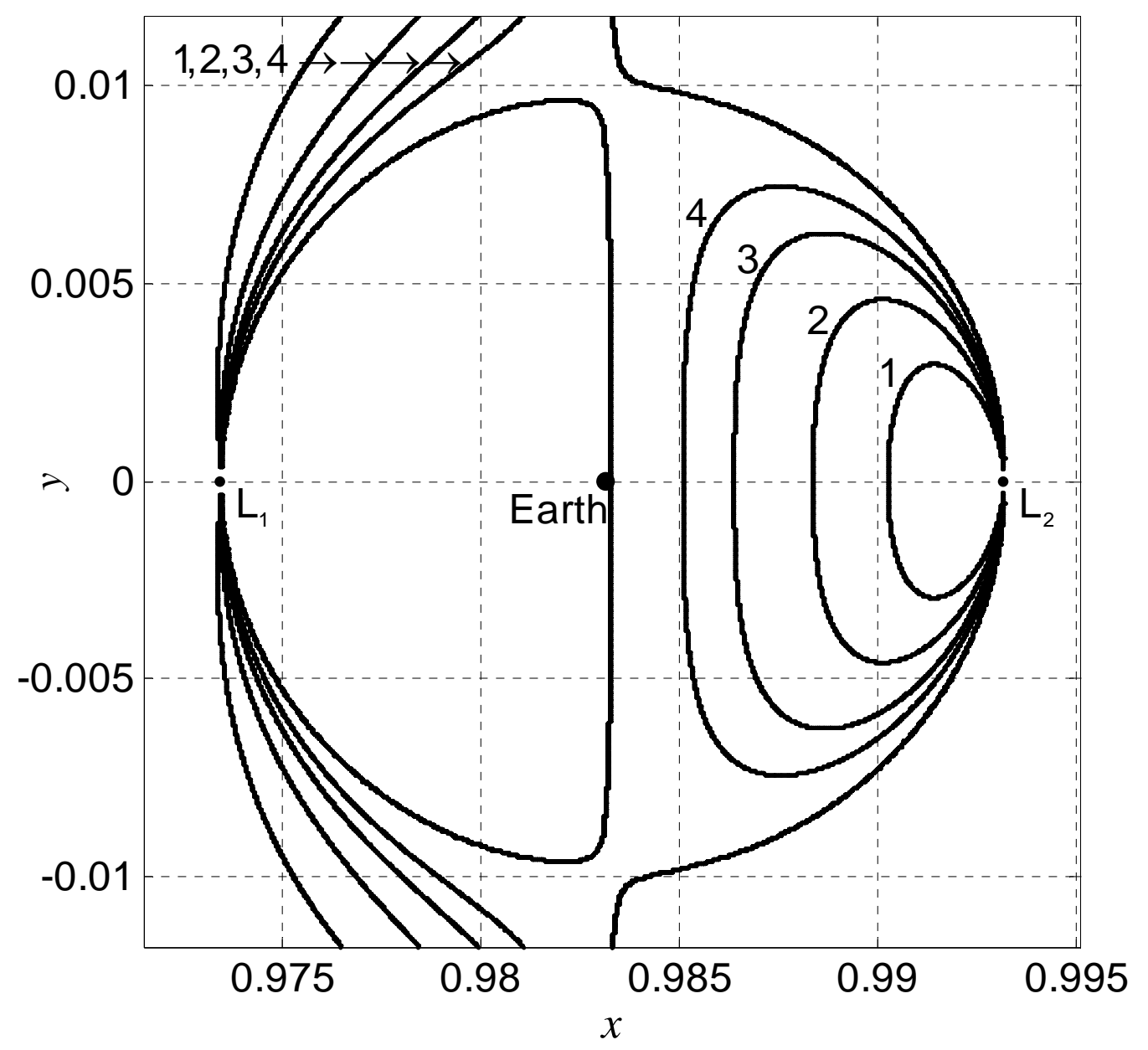


Figure 4 (a).

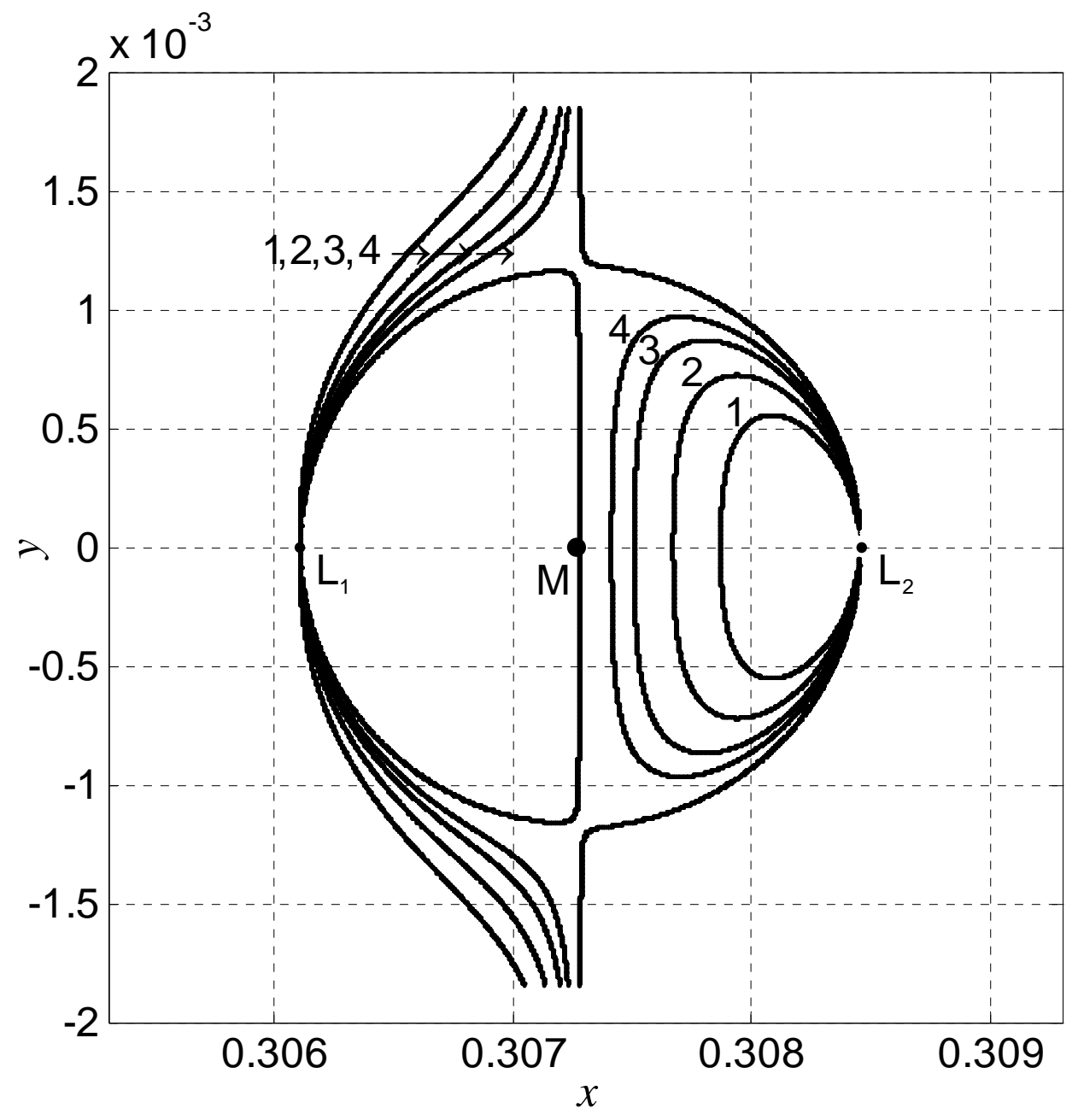


Figure 4(b).

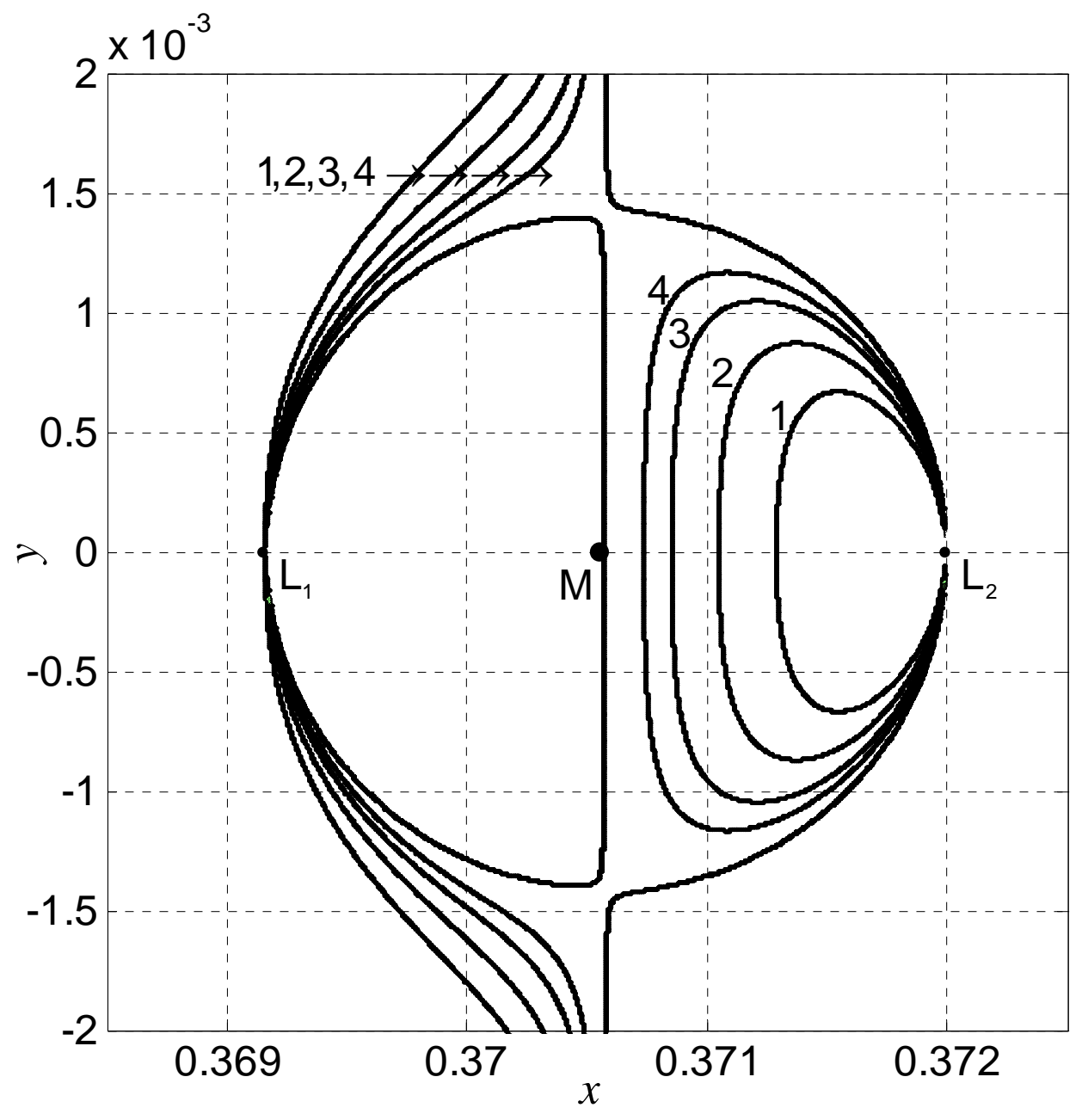


Figure 4 (c).

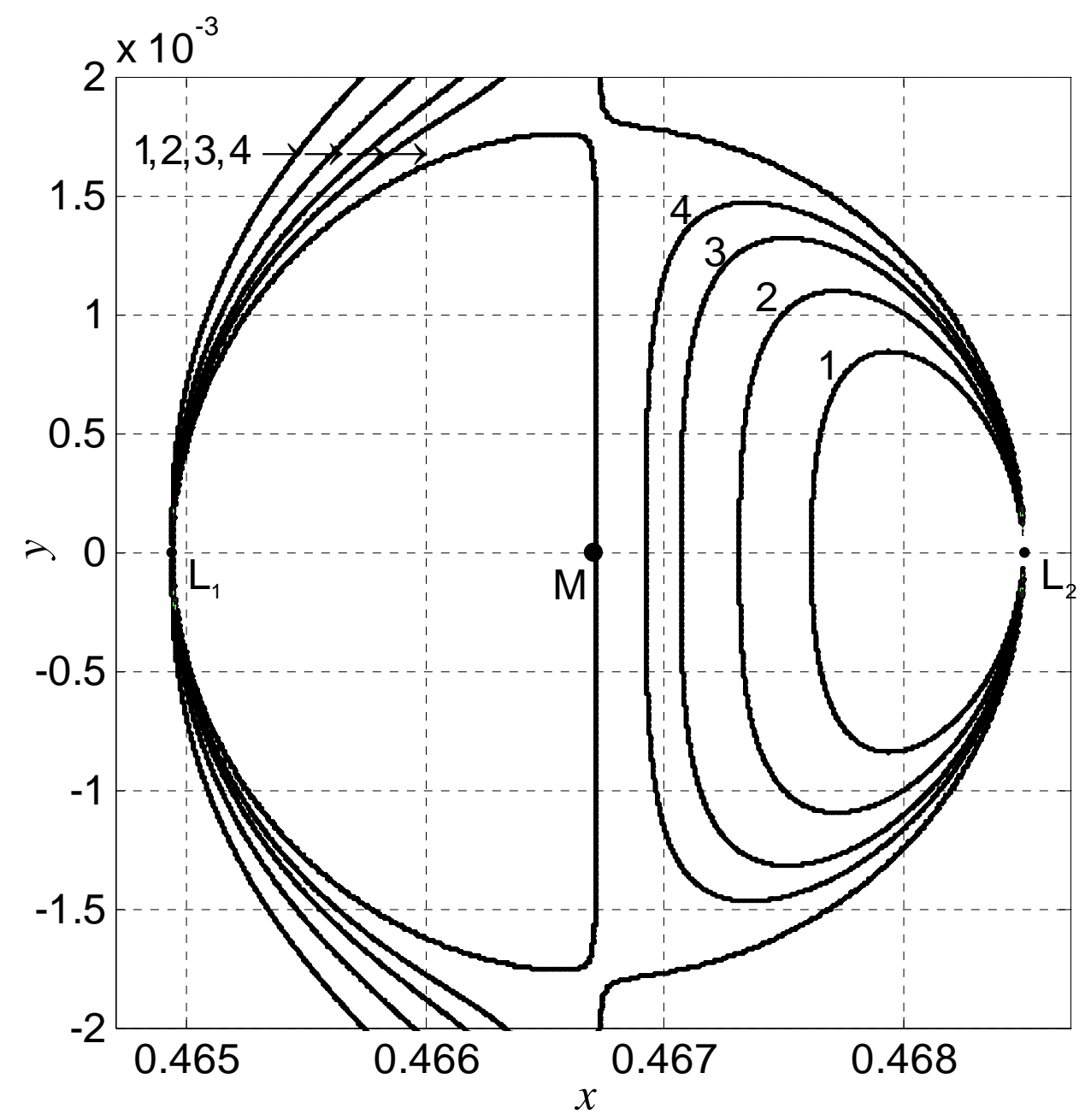


Figure 5.

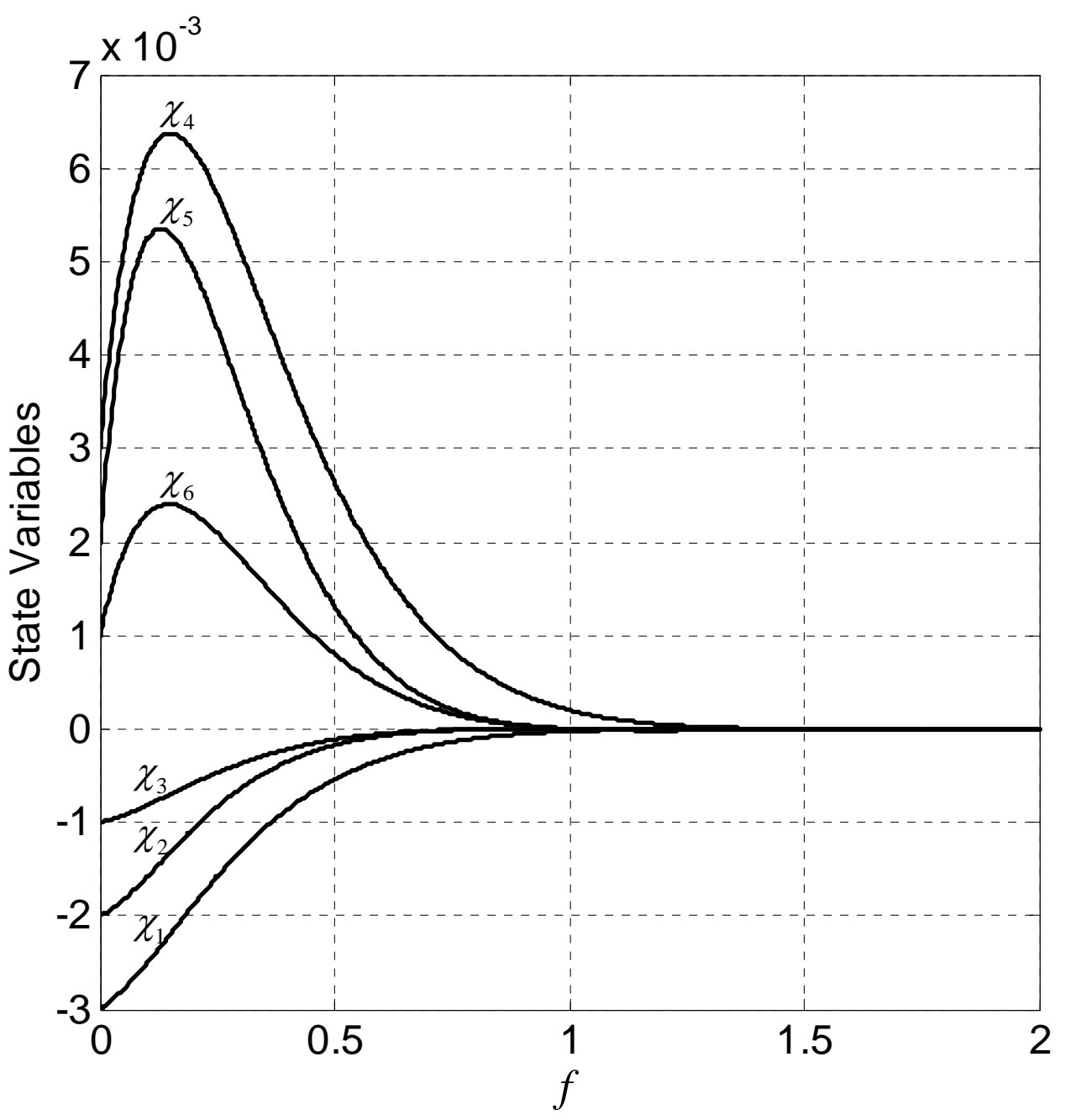

